\title{
Aplicação de ondas elementais no estudo de vibrações geradas por desmontes de rochas
}

\author{
Paulo José Costa Couceiro Júnior ${ }^{1 *}$ (1) \\ Eraldo Florêncio da Silva Júnior
}

\section{Resumo}

A aplicação de cargas explosivas sequenciadas para a escavação e fragmentação de rochas constitui uma operação unitária essencial na mineração. Entretanto, a liberação de energia resultante desse processo gera, entre outros fenômenos, um evento sísmico adverso que pode condicionar a viabilidade do projeto. Distintas técnicas de controle e previsão dos níveis de vibrações foram desenvolvidas nas últimas décadas, tais como a aplicação de ondas elementais na modelagem sísmica resultante de desmontes de rochas. A superposição de ondas individuais na conformação de histórias temporais das velocidades de partícula permite a verificação de distintos aspectos do desmonte, tais como o efeito dos tempos de retardo e sequenciamento das cargas explosivas, orientação da propagação das ondas sísmicas, e incertezas envolvidas no projeto, entre outros. Neste trabalho, um modelo de ondas elementais, baseado em variáveis estocásticas compatíveis com simulações Monte Carlo, é apresentado com o intuito de prever os níveis de vibrações mais prováveis de um desmonte de rochas. Finalmente, esta metodologia é exemplificada em um exemplo de aplicação, cujo níveis das vibrações necessitavam medidas preditivas mais fiáveis para garantir a viabilidade da obra.

Palavras-chave: Ondas elementais; Vibrações; Desmonte de rocha.

\section{Application of signature waves on the study of vibrations generated by rock blasting}

\begin{abstract}
The application of sequential explosive charges for excavation and fragmentation of rocks constitute an essential unit operation in mining. However, the liberation of energy resulting from this process creates, within other phenomena, an adverse seismic event that may condition the viability of the project. Different control and predicting vibration techniques have been developed in the last decades, such as the application of signature waves in modeling vibrations resulting from rock blasting. The superposition of individual waves for the conformation of particle velocities time-histories allows the verification of different blasting aspects, such as the effect of delays and sequence of detonation, orientation of the propagation path of waves, and uncertainties involved in the project, and others. In this work, a stochastic signature hole model is presented together with Monte Carlo simulations technique, which permits the prediction of the most probable vibration levels of a given blast. Finally, this methodology is illustrated in an example of application whose vibration levels required more reliable predictive measures to ensure the viability of the work.
\end{abstract}

Keywords: Signature wave; Vibrations; Rock blasting.

\section{Introdução}

Alguns dos primeiros estudos sobre a aplicação de ondas elementais na modelagem de vibrações geradas por desmontes de rochas podem ser encontrados nos trabalhos de Andrews [1], Anderson et al. [2,3] e Crenwelge [4], entre outros, como Hinzen et al. [5] ou Blair [6]. O uso de técnicas de superposição de ondas individuais, chamadas de ondas elementais, permitiu aprofundar o entendimento sobre a influência dos tempos de retardo e sequência de iniciação nas intensidades das vibrações [6-9] e frequências dominantes resultantes de um desmonte de rocha [7,10-13]. Como consequência, tornou-se possível mapeamento dos tempos de retardos ideais, devido a interferência destrutiva entre ondas e a formação de frequências dominantes preferentes, para atender os limites de vibrações aplicáveis ao projeto. Com o

${ }^{1}$ Advanced Applied Solutions Department, MAXAM, Madrid, Spain.

*Autor correspondente: paulocouceiro@gmail.com

2176-1523 (C) Couceiro Júnior et al. Publicado pela ABM. Este é um artigo publicado em acesso aberto (Open Access) sob a licença Creative Commons Attribution, que permite uso, distribuição e reprodução em qualquer meio, sem restrições desde que o trabalho original seja corretamente citado. 
advento dos detonadores eletrônicos, dotados de tempos de retardos super precisos, as técnicas de modelagem baseadas na convolução de ondas elementais ganharam grande relevância no campo de estudo das vibrações $[7,14,15]$.

O estudo de vibrações através de ondas elementais, portanto, é uma técnica de modelagem baseada na superposição de ondas individuais, produzidas por cargas sequenciadas, em um ponto concreto do espaço, após estas se propagarem pelo terreno [10]. Ainda que técnicas lineares e não lineares de superposição estejam disponíveis, a aproximação convolutiva linear continua sendo apropriada $[10,16]$ quando se estuda as vibrações geradas por desmontes de rocha em campo longínquo. O princípio básico desta técnica se sustenta no uso de uma onda elemental, gerada por uma única carga, para modelar a história temporal de vibrações através da convolução escalada de acordo com as cargas sequenciadas de um dado desmonte, em um ponto de interesse do terreno. Portanto, o modelo tem em conta a geometria das malhas de perfuração, cargas operantes, tempos e sequência de iniciação, dispersão dos tempos de retardos, velocidade sísmica do terreno, entre outros.

A implementação de um método de análise e modelagem de vibrações geradas por desmontes de rochas, fundamentada no conceito de ondas elementais, e sua aplicação em um estudo de caso, somam-se como objetivo deste trabalho. Ademais, a possibilidade de incorporar incertezas quanto ao tempo de retardo, características do terreno, erros de carga, entre outros, através das técnicas probabilísticas combinadas com simulações Monte Carlo [7,8,17], permite mapear todo um espectro de possíveis resultados, incrementando a fiabilidade das previsões em projetos onde os níveis de vibrações são críticos.

\section{Materiais e métodos}

\subsection{Fundamentos da análise com ondas elementais}

A análise de vibrações através das ondas elementais se fundamenta na superposição linear de pulsos vibratórios, escalados proporcionalmente à carga operante, para realizar a convolução dos sinais individuais gerados por cada furo de mina em um ponto concreto do espaço [8]. A composição final da história temporal [7], para um desmonte de produção de $N$ furos, pode ser representada através da seguinte Equação 1:

$$
\dot{u}(t)=S_{i}(t) \otimes \sum_{i=1}^{N} \alpha_{i} \delta\left(t-\Delta t_{i}\right)
$$

onde $\dot{u}(t)$ é a história temporal do evento sísmico simulada $(\mathrm{mm} / \mathrm{s}) ; \alpha_{i}$ é a amplitude da onda elemental $i(\mathrm{~mm} / \mathrm{s}) ; S_{i}(t)$ é a forma da onda elemental normalizada; $\delta\left(t-\Delta t_{i}\right)$ é a função delta de Dirac, representando o momento de iniciação da carga $i$, incluindo os efeitos de dispersão; $N$ é o número de furos de mina; $t$ é o tempo; $\otimes$ representa a operação de convolução dos sinais.

Adicionalmente, deve-se computar o tempo efetivo de chegada dos pulsos de ondas individuais, produzidas pela detonação de cada furo, a fim de convolucionar a contribuição de cada onda elemental. Assim, o tempo efetivo de chegada é composto pela soma do tempo de detonação e o de propagação. Esta relação $[7,8]$ pode ser escrita como (Equação 2):

$$
\left(t_{e}\right)_{i}=\left(t_{n}\right)_{i}+\frac{R_{i}}{C}
$$

onde $\left(t_{e}\right)_{i}$ é o tempo efetivo de chegada associado ao furo $i ;\left(t_{n}\right)_{i}$ é o tempo acumulado na sequência de detonação (incluindo a dispersão) da carga $i ; R_{i}$ é a distância entre o carga $i$ e o ponto de interesse; e $C$ é a velocidade sísmica do meio de propagação.

Por outro lado, a amplitude $\alpha_{i}$ da onda elemental $S_{i}(t)$ é calculada através da combinação de leis de atenuação do terreno com mecanismos atenuantes que podem afetar a plena conformação da intensidade vibratória, tais como os efeitos de confinamento e triagem (Equação 6). Cada um desses aspectos será brevemente revisado.

A abordagem estatística da lei de atenuação é uma das técnicas mais comuns para estudar a previsibilidade das vibrações. A estratégia do método consiste em ajustar uma curva em função da distância escalonada para um conjunto das velocidades pico de partícula experimentais. Portanto, uma função matemática, que envolva os parâmetros relevantes do problema, é necessária para realizar a regressão estatística. Esta função foi desenvolvida através de um método baseado na análise dimensional $[18,19]$ do problema, combinando variáveis dependentes e independentes associadas aos fenômenos sísmicos. Assim, a seguinte lei de atenuação, em função da distância escalonada quadrática [19], é adotada neste trabalho.

$$
P P V=K\left(\frac{D}{Q^{1 / 2}}\right)^{\beta}
$$

onde $P P V$ é velocidade pico de partícula $(\mathrm{mm} / \mathrm{s}) ; Q$ é a carga máxima por espera $(\mathrm{kg}) ; D$ é a distância $(\mathrm{m}) ; K$ e $\beta$ são constantes empíricas obtidas experimentalmente.

Entretanto, as amplitudes das ondas individuais sofrem perturbações devido às mudanças na qualidade do terreno presentes na direção de propagação das ondas sísmicas, ocasionadas pela presença de material fraturado, resultante de furos previamente detonados, entre outros fatores. Diversas soluções matemáticas foram propostas para tratar esses fenômenos [7,11]. A ideia básica assume a existência de um efeito de triagem em função do número de cargas previamente detonadas na direção de propagação das ondas geradas pelos furos restantes na sequência de detonação. Tendo esta consideração em mente, Blair [7] propôs a seguinte função de triagem: 


$$
f_{S}\left(N_{S}\right)=\frac{D^{2}}{D^{2}+g \sqrt{N_{S}}}
$$

onde $f_{S}\left(N_{S}\right)$ é a função de triagem; $g$ é uma constante ajustada experimentalmente; $D$ é a distância entre a carga detonante e o ponto de interesse; e $N_{S}$ é o número de furos de triagem.

No presente trabalho, o método de contagem dos furos de triagem $N_{S}$ é ligeiramente distinto daquele proposto por Blair [7] e Yang e Scovira [11]. Aqui se assume que todos os furos previamente detonados, que se encontram dentro de uma área elíptica formada entre a carga detonante e o ponto de interesse, são contabilizados como furos de triagem. A Figura la representa, de forma esquemática, a quantificação do número de furos detonados para a avaliação do efeito de triagem.

Adicionalmente, assume-se que a presença de material fraturado ao redor do furo que se encontra em processo de detonação afeta o seu grau de confinamento. Como consequência, acredita-se que uma consequente dissipação de energia, devido a um menor confinamento, possa afetar a intensidade vibratória resultante no ponto de interesse, ainda que o material fraturado não esteja na direção de propagação das ondas sísmicas, tal como apresentado na Figura 1b. Esse comportamento é modelado através da seguinte equação:

$$
f_{C}\left(N_{C}\right)=\frac{1}{\left(1+N_{C}\right)^{\gamma_{1}}}
$$

onde $f_{C}\left(N_{C}\right)$ é a função de confinamento e $N_{C}$ é número de furos detonados dentro da área de influência $R_{C}=3 / 2\left(B^{2}+S^{2}\right)^{0.5}$; $B$ e $S$ são os afastamento e espaçamento, respectivamente; $\gamma_{1}$ é uma constante obtida experimentalmente.

\section{(a)}

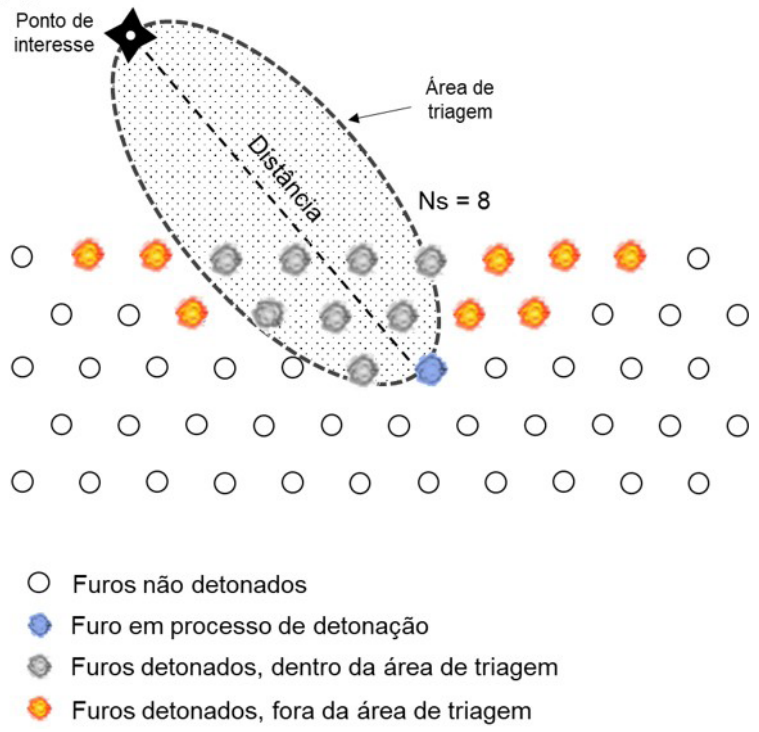

Finalmente, de forma similar à proposta por Blair [7], a amplitude $\alpha_{i}$ final aplicada a onda elemental normalizada $i$ pode ser estimada ao combinar as Equações 3, 4 e 5.

$$
\alpha_{i}=f_{S} f_{C} P P V_{i}
$$

onde $P P V_{i}$ é a velocidade pico de partícula associada ao furo $i$, calculada através da aplicação da Equação 3.

As histórias temporais, simuladas através da metodologia descrita nesse trabalho, permitem quantificar importantes propriedades relacionadas às vibrações, tais como a velocidade pico de partícula e a frequência dominante, fundamentais para contrastar as previsões com a normativa vigente.

Por outro lado, as incertezas das variáveis aleatórias envolvidas no problema, tais como carga por furo, distancias, dispersões de tempos, entre outros, podem ser introduzidas ao estudo através de métodos estatísticos como Monte Carlo, reproduzindo um elevado número de sucessivas simulações para calcular a probabilidade do fenômeno estudado.

\section{Resultados}

\subsection{Exemplo de aplicação}

Pretende-se exemplificar a metodologia apresentada neste trabalho com um desmonte de produção representativo do projeto de derrocagem e dragagem do Porto Sudeste, localizado na baía de Sepetiba, no Rio de Janeiro. Os resultados simulados serão comparados com medições reais em pontos específicos ao longo da malha de monitoramento sismográfico do projeto.

(b)

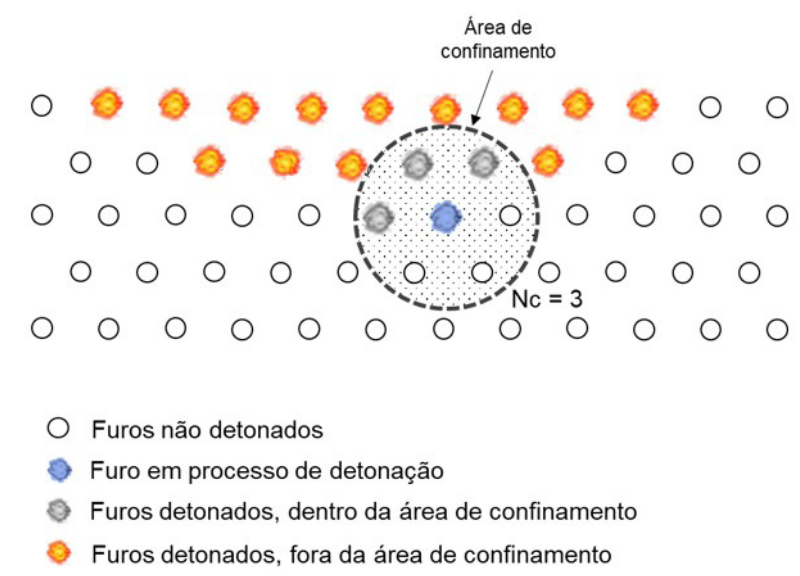

Figura 1. Representação esquemática usada para realizar a quantificação os efeitos de (a) triagem e (b) confinamento na conformação das intensidades vibratórias no processo de convolução de ondas elementais. 
O maciço rochoso estava composto de blocos naturais, cavernas e rocha pré-fraturada. Como consequência, foram aplicados procedimentos especiais de carga, com a utilização de camisas plásticas. A malha de perfuração foi $1.5 \times 2.3 \mathrm{~m}$ sobre uma espessura de rocha média de $2.5 \mathrm{~m}$. Um total de 90 furos, com diâmetro de $105 \mathrm{~mm}$, foram distribuídos ao longo de 8 filas e carregados com um explosivo bombeado. O consumo específico foi de $2.52 \mathrm{~kg} / \mathrm{m}^{3}$. Os tempos de retardos aplicados na temporização da detonação foram de $42 \mathrm{~ms}$ entre furos por $25 \mathrm{~ms}$ entre filas, com uma saída orientada por uma de suas esquinas.

\subsection{Dados experimentais}

Diversas ondas elementais foram medidas em diferentes distâncias e localizações durante cada uma das campanhas sismográficas. As distâncias variaram entre $132 \mathrm{~m}$ a $509 \mathrm{~m}$, para cargas de $15,25,40,30,50$ e $75 \mathrm{~kg}$. A Figura 2 apresenta duas das ondas elementais registradas, especificamente para as distâncias de $132 \mathrm{~m}$ e $401 \mathrm{~m}$. Assim, esta ampla amostragem de distâncias e pontos de monitoramento, permitiram selecionar as ondas elementais mais apropriadas e representativas durante o processo de análise das vibrações resultantes de detonações de produção.

As intensidades das vibrações registradas durante as campanhas sismográficas com ondas elementais permitiram a obtenção das leis de atenuação através de métodos de ajuste estatístico. A Figura 3 apresenta as amplitudes pico de partícula em função da distância escalonada para cada uma das direções de vibração (transversal, vertical, e longitudinal). As equações resultantes dessa análise são necessárias para a estimação dos valores de $P P V_{i}$ presente na Equação 3.

\subsection{Discussão de resultados}

Foram realizadas simulações das histórias temporais de vibrações para cada ponto de monitoramento sismográfico. A sequência de iniciação foi computada tendo em conta os retardos nominais, a amarração e o ponto de iniciação do desmonte. Uma vez que detonadores não elétricos foram usados, assumiu-se que a dispersão dos tempos de retardos segue uma distribuição normal, com um desvio padrão de $3 \%$. Silva Castro [8] investigou desvios padrões de detonadores não elétricos e eletrônicos, no qual detonadores de $25 \mathrm{~ms}$

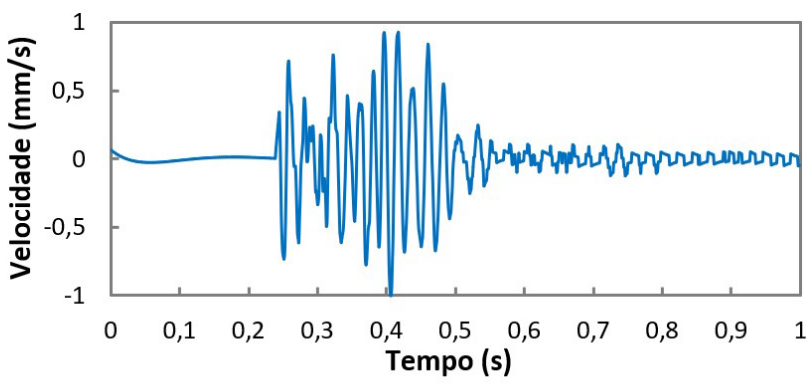

(b) Onda elemental P8 - 401m

Figura 2. Ondas elementais normalizadas (direção longitudinal), registradas a $132 \mathrm{~m}$ e $401 \mathrm{~m}$. Nota-se a transformação da forma da onda devido à complexidade envolvida durante a propagação das ondas em longas distâncias.

(a) Transversal

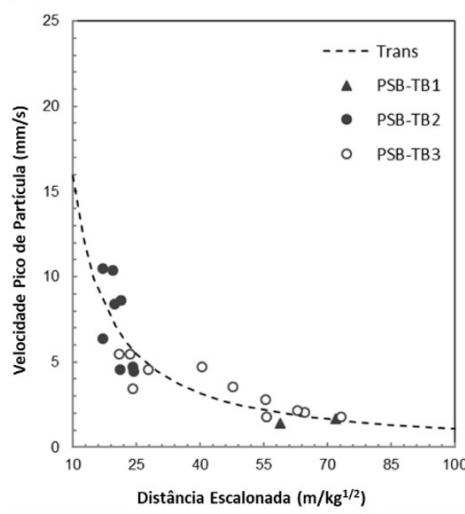

$P P V_{T}=232\left(\frac{D}{Q^{1 / 2}}\right)^{-1.164}$ (b) Vertical

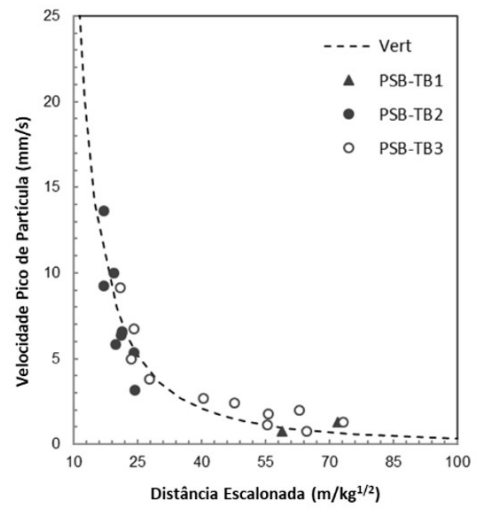

$P P V_{V}=2801\left(\frac{D}{Q^{1 / 2}}\right)^{-1.954}$ (c) Longitudinal

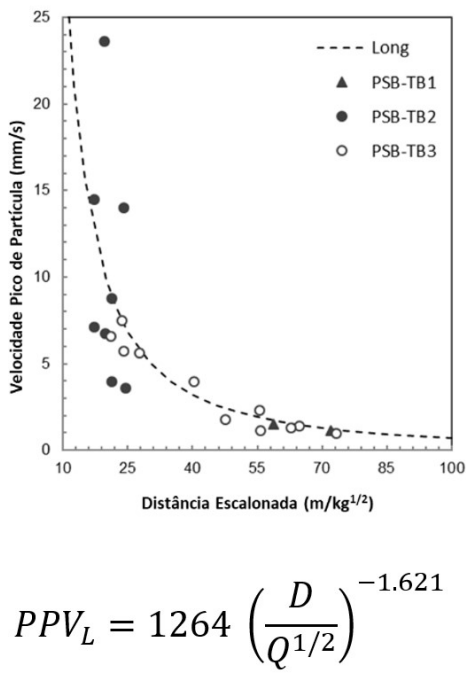

Figura 3. Leis de atenuação obtidas sobre os registros de ondas elementais para cada uma das direções do evento sísmico. 


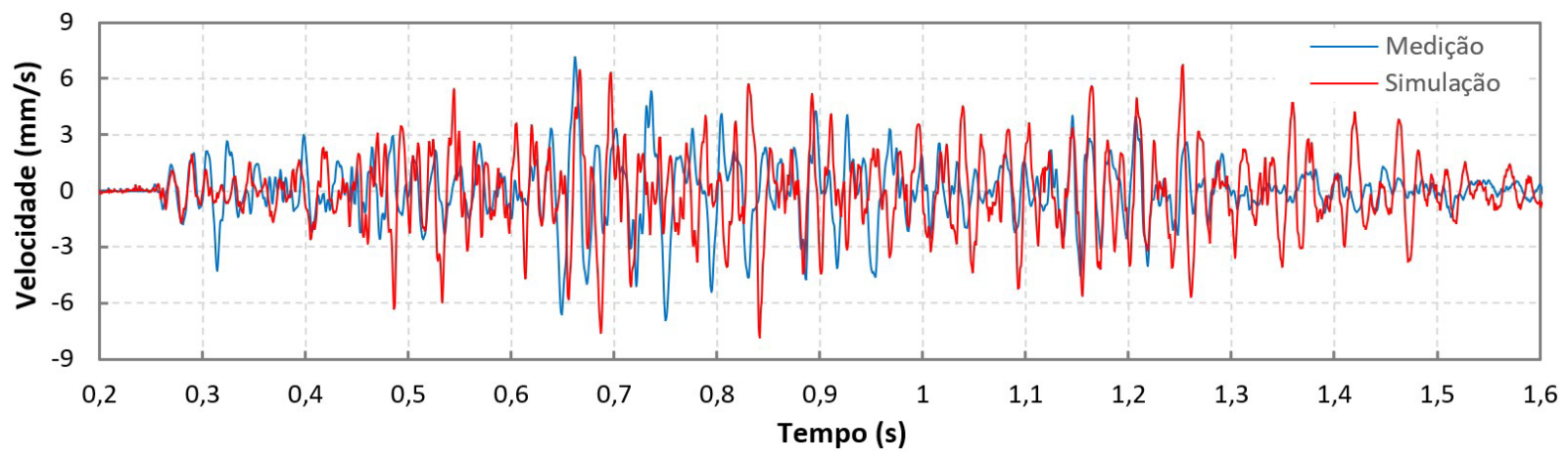

Figura 4. Histórias temporais de vibrações simulada (vermelho) e real (azul), registrada a uma distância de $116 \mathrm{~m}$ da detonação.
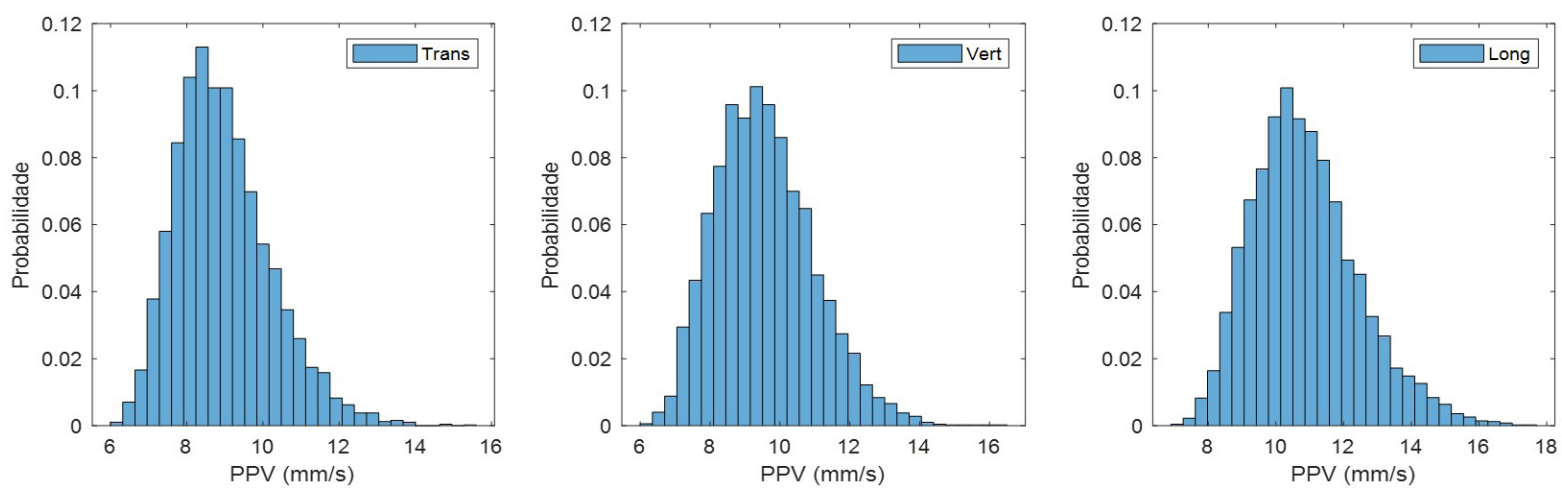

Figura 5. Probabilidades de ocorrência de velocidades pico de partícula (PPV) a uma distância de 116m da detonação, simuladas com Monte Carlo (5000 simulações).

apresentaram desvios ao redor de $3 \%$. Ademais, em base as cartas sísmicas do projeto, assumiu-se uma velocidade sísmica média do meio de propagação de $3500 \mathrm{~m} / \mathrm{s} \pm 50 \mathrm{~m} / \mathrm{s}$. Por outro lado, dada a complexidade do processo de carga, tem-se que as cargas explosivas unitárias possam ter tido variações de até $10 \%$ no cômputo de quantidades.

Inicialmente, apresenta-se na Figura 4 o resultado de uma dessas simulações, em concreto, a do ponto P2, localizado a 116m da detonação, em comparação com o registro real do evento. Esta simulação representa a vibração esperada na direção transversal. Considerando as complexidades do problema, como variabilidades geológicas, dispersão dos tempos de retardos, entre outros, o resultado simulado se correlaciona relativamente bem com a história de vibração temporal real. $\mathrm{O}$ valor da velocidade pico de partícula simulado neste ponto em particular foi de $7.81 \mathrm{~mm} / \mathrm{s}$ enquanto o real foi de $7.12 \mathrm{~mm} / \mathrm{s}$.

Entretanto, uma vez que uma única simulação não reflete a variabilidade estatística resultante das dispersões dos tempos de retardos e velocidade sísmica do terreno, ou outras incertezas, realizaram-se simulações Monte Carlo com 5000 interações $^{1}$, a fim de prever as velocidades pico de

I Este número de interações foi escolhido para se conseguir uma distribuição de probabilidade mais suave, uma vez que inúmeras combinações aleatórias são possíveis como resultado das dispersões dos tempos de retardos e dos tempos de propagação, com relação à superposição construtiva ou destrutiva entre os picos das ondas elementais geradas por cada furo. partícula mais prováveis em cada ponto de monitoramento sismográfico. A Figura 5 mostra as simulações resultantes no ponto P2. Neste caso, as vibrações foram $8.81 \pm 1.61 \mathrm{~mm} / \mathrm{s}$, $9.45 \pm 1.80 \mathrm{~mm} / \mathrm{s}$ e $10.68 \pm 2.01 \mathrm{~mm} / \mathrm{s}$, com margem de erro estabelecido em $80 \%$ do intervalo de confiança, para as direções transversal, vertical e longitudinal, respectivamente. As velocidades pico de partícula reais, registradas após a detonação do desmonte de produção, foram $7.12 \mathrm{~mm} / \mathrm{s}$, $9.20 \mathrm{~mm} / \mathrm{s}$ e $10.35 \mathrm{~mm} / \mathrm{s}$, que são congruentes com as simulações realizadas.

Os resultados das simulações Monte Carlo em todos os pontos de monitoramento, juntamente com os valores medidos e previstos com a aplicação direta de leis de atenuação, para cada uma das três direções da vibração, são apresentados na Figura 6. Observa-se uma melhor correlação entre os resultados obtidos com técnicas de ondas elementais frente às obtidas com as leis de atenuação. Adicionalmente, a Tabela 1 apresenta os vetores resultantes de velocidades de partícula das máximas vibrações das componentes transversal, vertical e longitudinal, e os respectivos erros relativos e médios totais resultantes das análises aplicadas neste estudo. Observa-se que o erro médio total para o caso das ondas elementais foi de $8.0 \%$ enquanto as resultantes da aplicação direta de leis de atenuação foram de 18.1\%.

Esses resultados indicam como as técnicas de análise de vibrações com ondas elementais podem ser mais precisas quando comparadas com técnicas tradicionais. 
(a) Transversal

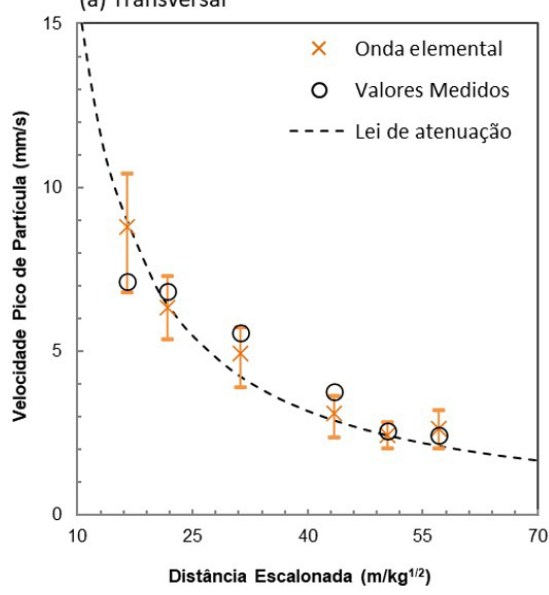

(b) Vertical

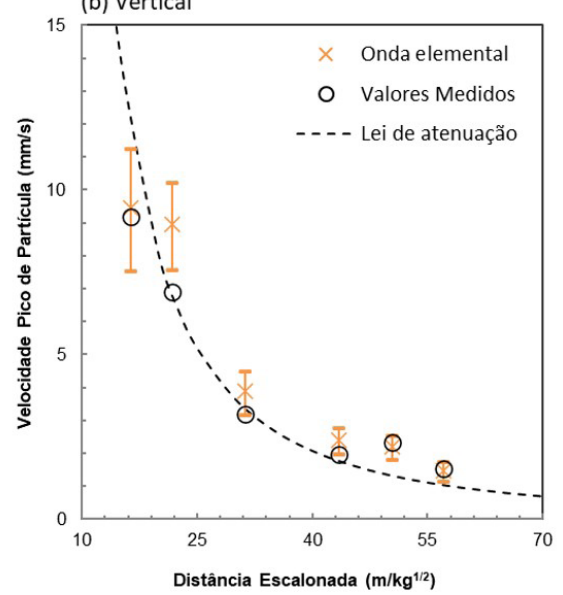

(c) Longitudinal

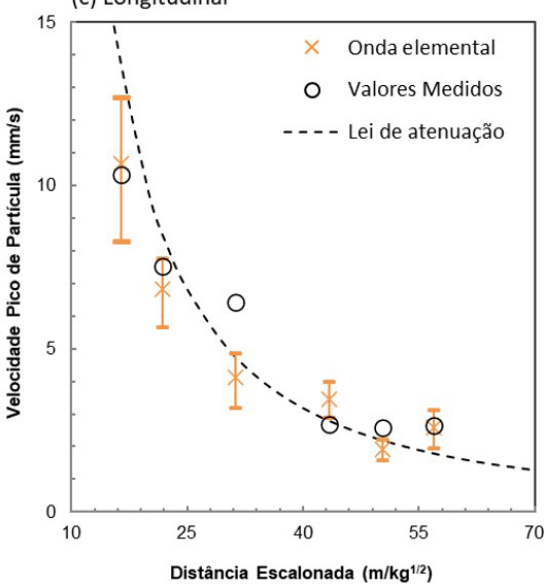

Figura 6. Velocidades pico de partícula em função da distância escalonada resultantes das simulações Monte Carlo, leis de atenuação e medidas em distintos pontos ao longo da malha de monitoramento sismográfico.

Tabela 1. Resultados medidos e simulados com leis de atenuação e simulações Monte Carlo (5000 interações) e seus respectivos erros relativos e médios total

\begin{tabular}{|c|c|c|c|c|c|c|}
\hline \multirow[t]{2}{*}{ Métodos } & \multicolumn{6}{|c|}{ Pontos de Monitoramento } \\
\hline & $\mathbf{P 2}$ & P3 & P5 & P6 & P7 & P8 \\
\hline Valores medidos $(\mathrm{mm} / \mathrm{s})$ & 15,57 & 12,28 & 9,08 & 5,03 & 4,32 & 3,91 \\
\hline Lei de atenuação $(\mathrm{mm} / \mathrm{s})$ & 20,10 & 12,69 & 7,20 & 4,39 & 3,53 & 2,95 \\
\hline Erro relativo \% & $29,1 \%$ & $3,3 \%$ & $-20,7 \%$ & $-12,8 \%$ & $-18,2 \%$ & $-24,4 \%$ \\
\hline Erro médio total \% & \multicolumn{6}{|c|}{$18,1 \%$} \\
\hline Onda elemental $(\mathrm{mm} / \mathrm{s})$ & 16,76 & 12,92 & 7,51 & 5,21 & 3,79 & 3,98 \\
\hline Erro relativo \% & $7,7 \%$ & $5,1 \%$ & $-17,4 \%$ & $3,6 \%$ & $-12,2 \%$ & $1,8 \%$ \\
\hline Erro médio total \% & \multicolumn{6}{|c|}{$8,0 \%$} \\
\hline
\end{tabular}

\section{Conclusões}

Um modelo preditivo baseado na aplicação de ondas elementais para o controle dos níveis de vibrações gerados por desmontes de rochas foi apresentado. O modelo é sensível a importantes parâmetros do projeto, tais como cargas individuais dos furos, temporização da sequência de detonação, propriedades do meio de propagação, incertezas do projeto como dispersão dos tempos de retardos, entre outros. Ao introduzir o efeito da perda de confinamento na conformação da intensidade das amplitudes vibratórias, o modelo permite, além do efeito de triagem, inferir as vibrações de forma mais realista. Finalmente, no exemplo de aplicação apresentado, o emprego de técnicas baseadas em ondas elementais permitiram obter melhores estimativas das velocidades pico de partícula quando comparadas com técnicas tradicionais de leis de atenuação, uma vez que tanto a geometria do fogo quanto a sequência de iniciação são consideradas no estudo. Contudo, as premissas fundamentais do modelo, assim como a coerência no processo de obtenção de dados experimentais, devem ser levadas em consideração quando de sua aplicação no dimensionamento de projetos de desmontes de rochas em circunstâncias de alta complexidade técnica.

\section{Referências}

1 Andrews AB. Design criteria for sequential blasting. In: Proceedings of the 7th Annual Conference on Explosives and Blasting Techniques; 1981 January 19-23; Phoenix, United States. Phoenix: Society of Explosives Engineers; 1981. p. 173-192.

2 Anderson D, Winzer SW, Ritter A. Synthetic delay versus frequency plots for predicting ground vibration from blasting. In: Proceedings of the of the 3rd International Symposium on Computer-Aided Seismic Analysis and Discrimination; 1983 June 15-17; Washington, United Sates. Los Angeles: IEEE Computer Society; 1983. p. 70-74.

3 Anderson D, Winzer SW, Ritter AP, Reil JW. A method for site-specific prediction and control of ground vibration from blasting. In: Proceedings of the 11th Annual Conference on Explosives and Blasting Techniques; 1985 January 27-February 1; San Diego, United States. San Diego: Society of Explosives Engineers; 1985. 
Aplicação de ondas elementais no estudo de vibrações geradas por desmontes de rochas

4 Crenwelge OEJ. Use of single charge vibration data to interpret explosive excitation and ground transmission characteristics. In: Proceedings of the 14th Annual Conference on Explosives and Blasting Techniques; 1985 January 31-February 5; Anaheim, United Sates. Anaheim: Society of Explosives Engineers; 1988. p. 151-160.

5 Hinzen K, Lüdeling R. A new approach to predict and reduce blast vibration by modeling of seismograms and using a new electronic initiation system. In: Proceedings of the 13th Annual Conference on Explosives and Blasting Techniques; 1987 February 2-5; Miami, United States. Miami: Society of Explosives Engineers; 1987. p. 144-161.

6 Blair DP. The measurement, modelling and control of ground vibrations due to blasting. In: Proceedings of the Second International Symposium on Rock Fragmentation by Blasting; 1987 August 23-26; Keystone, United Sates. Bethel: Society for Experimental Mechanics; 1987. p. 88-101.

7 Blair DP. Statistical models for ground vibration and airblast. International Journal for Blasting and Fragmentation. 1999;3(4):335-364.

8 Silva-Castro JJ. Blast vibration modeling using improved signature hole technique for bench blast [thesis]. Kentucky: College of Engineering, University of Kentucky; 2012.

9 Silva-Castro JJ. A different methodology to control and predict ground vibrations from mine blasting. Canadian Geotechnical Journal. 2019;56(7):929-941.

10 Spathis AT. A brief review of the measurement, modelling and management of vibrations produced by blasting. In: FRAGBLAST. Proceeding of the 9th International Symposium on Rock Fragmentation by Blasting; 2009 September 13-17; Granada, Spain. Granada: CRC Press; 2010; p. 1-11.

11 Yang R, Scovira D. A model for near and far field blast vibration based on multiple seed waveform and transfer functions. International Journal for Blasting and Fragmentation. 2010;4(2):91-116.

12 Silva-Castro JJ, Li L. Deconvolution of blast vibration signals by wiener filtering. Inverse Problems in Science and Engineering. 2018;26(10):1522-1538.

$13 \mathrm{Li} \mathrm{L}$. Synthesis of single-hole vibration waveforms from a mining blast [thesis]. Kentucky: College of Engineering, University of Kentucky; 2018.

14 Anderson DA, Brinckerhoff P. Signature hole blast vibration control: twenty years hence and beyond. In: Proceedings of the 34th Annual Conference on Explosives and Blasting Techniques; 2008 January 27-30; New Orleans, United Sates. New Orleans: International Society of Explosives Engineer; 2008. p. 1-12.

15 Agrawal H, Mishra AK. An innovative technique of simplified signature hole analysis for prediction of blastinduced ground vibration of multi-hole/production blast: an empirical analysis. Natural Hazards. 2020;100:111-132.

16 Blair DP. Some problems associated with standard charge weight vibration scaling laws. In: Proceeding of the 3rd International Symposium no Rock Fragmentation by Blasting; 1990 August 26-31; Brisbane, Australia. Brisbane: The Australasian Institute of Mining and Metallurgy; 1990. p. 149-158.

17 Blair DP. Vibration modelling and mechanisms for wall control blasting. In: Proceeding of the 12th International Symposium on Rock Fragmentation by Blasting; 2018 June 11-13; Luleå, Sweden. Luleå: Luleå University of Technology; 2018. p. 269-280.

18 Ambreseys NN, Hedron AJ. Dynamic behavior of rock masses. In: Stagg KC, Zienkiewicz O, editores. Rock mechanics in engineering practice. London: John Wiley \& Sons; 1968.

19 Dowding CH. Blast vibration monitoring and control. Englewood Cliffs: Prentice-Hall; 1985.

Recebido em: 27 Dez. 2019

Aceito em: 13 Set. 2020 